EPJ Web of Conferences 57, 01004 (2013)

DOI: $10.1051 /$ epjconf/20135701004

(C) Owned by the authors, published by EDP Sciences, 2013

\title{
Tunable gauge potential for spinless particles in driven lattices
}

\author{
J. Simonet ${ }^{1, a}$, J. Struck ${ }^{1}$, M. Weinberg ${ }^{1}$, C. Ölschläger ${ }^{1}$, P. Hauke ${ }^{2}$, \\ A. Eckardt ${ }^{3}$, M. Lewenstein ${ }^{2}$, K. Sengstock ${ }^{1}$ and P. Windpassinger ${ }^{1}$ \\ ${ }^{1}$ Institut für Laserphysik, Universität Hamburg, Hamburg, Germany \\ ${ }^{2}$ Institut de Ciències Fotòniques, Barcelona, Spain \\ ${ }^{3}$ Max-Planck-Institut für Physik komplexer Systeme, Dresden, Germany
}

\begin{abstract}
We present a universal method to create a tunable, artificial vector gauge potential for neutral particles trapped in an optical lattice. A suitable periodic shaking of the lattice allows to engineer a Peierls phase for the hopping parameters. This scheme thus allows one to address the atomic internal degrees of freedom independently. We experimentally demonstrate the realisation of such artificial potentials in a 1D lattice, which generate ground state superfluids at arbitrary non-zero quasimomentum [4].

This scheme offers fascinating possibilities to emulate synthetic magnetic fields in 2D lattices. In a triangular lattice, continuously tunable staggered fluxes are realised. Spontaneous symmetry breaking has recently been observed for a $\pi$-flux [23]. With the presented scheme, we are now able to study the influence of a small symmetry breaking perturbation.
\end{abstract}

\section{INTRODUCTION}

Emulation of gauge fields for ultracold atoms provides access to a class of exotic states arising in strong magnetic fields [1, 2]. Standard electromagnetic fields indeed do not couple to the motional degrees of freedom of chargeless atomic species investigated in optical lattices.

In quantum mechanics, a vector gauge potential impacts onto the kinetic part of the Hamiltonian. For systems discrete in space, its effects can be absorbed into a complex hopping parameter $J=|J| e^{i \theta}$ as depicted in Figure 1(a), where $\theta$ is a directional phase factor, called the Peierls phase. This can be easily apprehended via the example of the Aharonov-Bohm phase acquired by a charged particle when it travels along a closed contour pierced by a magnetic field [3]. Therefore, the quest for artificial magnetism is to realise situations where a neutral particle acquires a geometrical phase when it follows a closed contour.

We experimentally demonstrate the realisation of complex tunneling matrix elements in periodically forced optical lattices [4]. Jiménez-García et al. achieved a similar result by using a combination of Raman lasers and radio frequency fields in order to simultaneously create a state-dependent optical lattice and modify the hopping parameters [5]. On the contrary, our scheme does not impose any requirements onto the internal structure of the atoms since it relies on inducing a time periodic inertial force by accelerating the optical lattice.

In the first part, the generation of tunable Peierls phases via a suitable periodic forcing will be demonstrated in a one-dimensional lattice. The second part will concentrate on the extension of this scheme to generate staggered magnetic fluxes in a two-dimensional (2D) triangular lattice. Especially

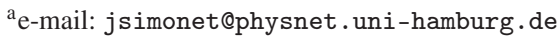

This is an Open Access article distributed under the terms of the Creative Commons Attribution License 2.0, which permits unrestricted use, distribution, and reproduction in any medium, provided the original work is properly cited. 
(a)

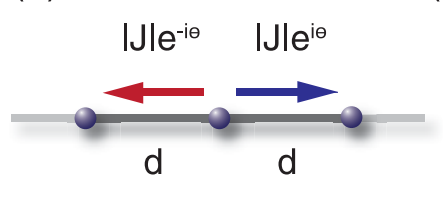

(b)

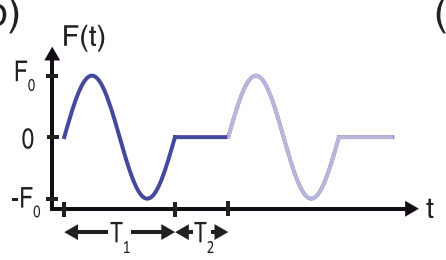

(c)

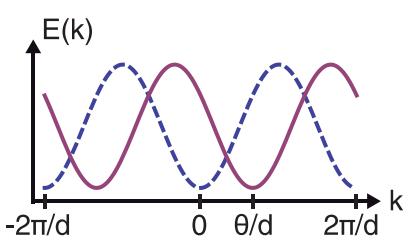

Figure 1. Periodic driving of the 1D lattice. (a) Realised complex tunneling elements on a 1D lattice, with spacing $d$. (b) Inertial force $\mathbf{F}$ of zero mean value resulting from the real space lattice periodic driving. $T_{1} / T_{2}$ is the asymmetry parameter. (c) Effective single-particle dispersion relation. The presence of a Peierls phase $\theta$ induces a shift of the dispersion relation minimum towards $\theta / d$ (solid line). As a reference, the dashed line represents the dispersion relation in absence of Peierls phase.

interesting is the regime of large flux strengths, where frustration - reflected by a two-fold degenerate ground-state - plays a key role.

\section{EXPERIMENTAL CONTROL OVER THE COMPLEX TUNNELING PARAMETERS}

Ultracold atoms confined in time-periodically driven optical lattices raise a large interest since the prediction of a metal-insulator like transition induced by a time-periodic driving force [6]. Several experimental groups have investigated the dynamical behaviour of non-condensed cold atoms $[7,8]$ or Bose-Einstein condensates $[9,10]$ under the combined effect of both a homogeneous time-independent and a time-periodic driving force. For a sinusoidal driving of an optical lattice, dynamical tunneling suppression and sign inversion of the tunneling matrix element have been reported [11, 12], leading to the coherent control of the superfluid-to-Mott insulator transition [13].

Here we show how to engineer complex tunneling matrix elements using a non-sinusoidal periodic forcing. This constitutes an essential prerequisite for the realisation of artificial gauge fields for uncharged particles [14].

\subsection{Periodical lattice forcing and renormalisation of the tunneling matrix elements}

The physics of ultracold bosons in the tight-binding regime of a lattice is governed by the Bose-Hubbard Hamiltonian:

$$
H=-J \sum_{\langle i, j\rangle} \hat{a}_{i}^{+} \hat{a}_{j}+\frac{U}{2} \sum_{i} \hat{n}_{i}\left(\hat{n}_{i}-1\right)
$$

where $\hat{a}_{i}\left(\hat{a}_{i}^{+}\right)$is a annihilation (creation) operator for a boson on the site $i$, and $\hat{n}_{i}$ denotes the corresponding number operator. The sum runs over all lattice sites and $\langle i, j\rangle$ denotes nearest neighbour sites. In the following, we consider a system in the weakly interacting regime where $U / J \ll 1$, with $J$ quantifying the tunneling amplitude and $U$ the on-site interaction energy.

Ultracold atoms in optical lattices, which are periodically forced with frequencies on the order of a few to tens of kilohertz, might appear as typical examples of non-equilibrium systems. On the contrary, Floquet theory allows to demonstrate that under high frequency periodic driving, the driven system behaves as the undriven system, but with renormalised matrix element $J^{\text {eff }}$ [15]. This renormalisation results from a time-averaging of the fast dynamics occuring in the lattice band-structure. As the only prerequisite, the modulation frequency has to be large with respect to the tunneling frequency but small compared to band excitation energies. In this regime, one can show that the driven system is to good 
approximation described by the effective time-independent Hamiltonian [16]:

$$
H_{\mathrm{eff}}=-\sum_{\langle i, j\rangle}\left|J_{i j}^{\mathrm{eff}}\right| e^{i \theta_{i j}} \hat{a}_{i}^{+} \hat{a}_{j}+\frac{U}{2} \sum_{i} \hat{n}_{i}\left(\hat{n}_{i}-1\right)
$$

An homogeneous inertial force $\mathbf{F}=-m_{R b} \ddot{\mathbf{x}}$ which is created by shaking the lattice along the periodic orbit $\mathbf{x}(t)$ in space gives rise to complex tunneling parameters $\left|J_{i j}^{\text {eff }}\right| e^{i \theta_{i j}}=\left\langle e^{i\left(\chi_{j}-\chi_{i}\right) / \hbar}\right\rangle_{T}$, where $\chi_{i}(t)=$ $\int_{t_{0}}^{t} d t^{\prime} \mathbf{r}_{i} . \mathbf{F}\left(t^{\prime}\right)-\left\langle\int_{t_{0}}^{t} d t^{\prime} \mathbf{r}_{i} \cdot \mathbf{F}\left(t^{\prime}\right)\right\rangle_{T}$.

The phase factors are non-trivial provided the forcing time-dependency breaks the two following symmetries: (a) reflection symmetry for a suitable time $\tau$, i.e. $v_{i}(t-\tau)=v_{i}(-t-\tau)$ and (b) shift (anti)symmetry, i.e. $v_{i}(t-T / 2)=-v_{i}(t)$. This requires forcing with more than one frequency component.

Experimentally, the one-dimensional lattice is generated by two independent, phase stabilised laser beams at $\lambda=830 \mathrm{~nm}$. The trapped ${ }^{87} \mathrm{Rb}$ atoms are accelerated along the lattice axis by modulating the frequency of one of the lattice beams with an acousto-optical modulator, a technique which is experimentally straightforward. As reported in Fig. 1(b), the forcing function used in our experiment is comprised of a train of sinusoidal pulses separated by periods with no forcing of periodicity $T=T_{1}+T_{2}=1 \mathrm{~ms}$. The zero mean value of this force prevents the transfer of a net-acceleration to the lattice. This leads to the following renormalised tunneling matrix element:

$$
\frac{J^{\mathrm{eff}}(K)}{J}=\frac{T_{2}}{T} e^{i K \frac{T_{1}}{T}}+\frac{T_{1}}{T} J_{0}(K) e^{-i K \frac{T_{2}}{T}}
$$

where $T_{1} / T_{2}$ is the asymmetry parameter and $J_{0}$ the zero-order Bessel function. The forcing parameter $K$ is defined as $K=m d^{2} v / \hbar$, with $v$ the amplitude of the frequency modulation, ranging here from 0 to $30 \mathrm{kHz}$.

For $T_{2} / T \rightarrow 0$, one recovers the harmonic driving which only allows to control the magnitude and sign of $J^{\text {eff }}$, as already investigated in $[11,12]$. In the opposite limit of small $T_{1} / T$, the tunneling amplitude $\left|J^{\text {eff }}\right|$ is only marginally affected whereas the phase depends linearly on the forcing amplitude $K$. In order to avoid unwanted excitations of the system induced by strong forcing, an intermediate value $T_{1} / T_{2}=2.1$ has been chosen for the experiment realised here. This induces a Peierls phase increasing monotoneously with the amplitude of the force.

\subsection{Detection of the Peierls phase}

The engineered complex phase of the hopping parameters manifests itself via a shift of the singleparticle dispersion relation by $\theta / d$ as depicted in Figure 1(c). It follows directly from the effective Hamiltonian (2), whose eigenstates are Bloch waves with the following dispersion relation:

$$
E(k)=-2\left|J^{\mathrm{eff}}\right| \cos (k d-\theta) \text {. }
$$

Therefore, this allows for the generation of superfluid ground-states at finite and tunable quasimomentum $k=\theta / d$ for which the group velocity $v_{g}=d E / d k$ is zero. Experimentally, we observe the relaxation of the condensate quasimomentum towards the minimum of the effective dispersion relation (see [4] for more details). Hence, the imprinted Peierls phase can be read out directly from the quasimomentum distribution revealed in time-of-flight after a sudden switch off of the lattice and the external potential.

As a central result Fig. 2(a) shows the experimental data together with the theoretical predictions from equation (3). After increasing the forcing amplitude slowly to the desired value, the corresponding quasimomentum distribution is recorded. From the obtained time-of-flight images, examples of which are shown in Fig. 2(b), we extract the Peierls phases $\theta$. The excellent agreement between experiment 
(a)

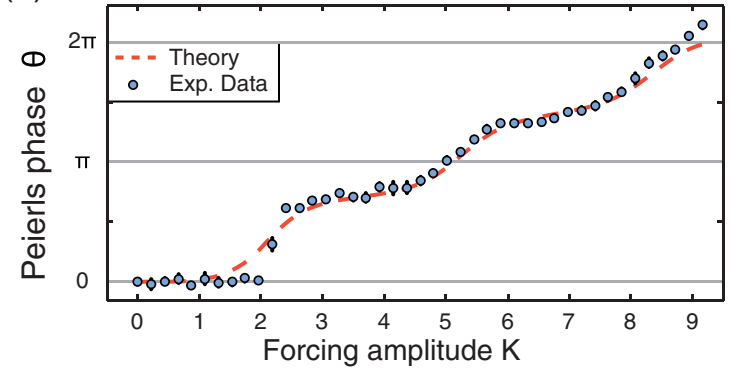

(b)

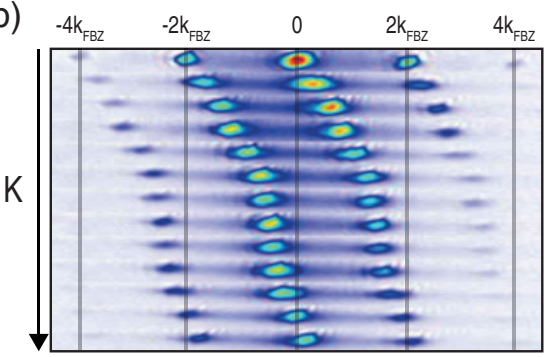

Figure 2. Creation of complex tunneling matrix elements. (a) The measured Peierls phases in a 1D driven optical lattice for different values of the forcing amplitude $K$ are depicted as circles. The dashed red curve corresponds to the theoretically expected values (equation (3)). (b) Quasimomentum distribution of the BEC after 27 ms time-offlight for different values of $K$. The Peierls phase as a function of $K$ is deduced from the observed shifts of the interference patterns.

and theory proves the controlled generation of Peierls phase $\theta \in[0,2 \pi]$. In addition, the experimental images demonstrate the large degree of coherence maintained in the atomic sample throughout the shaking process.

\section{STAGGERED MAGNETIC FLUXES IN A TRIANGULAR LATTICE}

Recently, several experimental ways have led to the realisation of artificial magnetic fields for ultracold atoms. The applied methods vary from trap rotation [17] to Berry phase imprinting either in a continuous system [18, 19] or in optical lattices [20, 23] (for a review see [21]). Such artificial fields have a strong impact on the single particle dispersion relation and result in cyclotron-like mass currents in discrete systems or the creation of vortices in a bulk. Additional phenomena arise in the presence of large amplitude staggered fluxes, where frustration plays a key role.

The technique employed here to engineer complex hopping parameters can be easily scaled to lattices of higher dimensions. We report on the experimental realisation of staggered fluxes in a 2D triangular lattice.

\subsection{Forced triangular lattice}

A two-dimensional triangular lattice is created by interfering three phase-locked laser beams in the $x y$ plane [22]. Our system, well within the weakly interacting regime, consists of an array of superfluid tubes arranged on a triangular pattern. The lattice is accelerated along a closed two-dimensional orbit by frequency modulating two of the three laser beams. The time-averaged effect of the inertial force leads to the renormalisation of all tunneling parameters. Varying the relative amplitudes of the forcing along $x$ and $y$ results in the independent control of two Peierls phases $\theta$ and $\theta^{\prime}$ (see Fig. 3(a)).

The total phase accumulated by the wavefunction after completing a loop around a plaquette is a gauge invariant quantity, called gauge flux. Homogeneous forcing, implemented by lattice shaking, cannot give rise to a non trivial flux through plaquettes with pairwise parallel edges like square or honeycomb. As a consequence, the total flux through the unit cell of the triangular lattice must be zero. However a non-zero flux through a single triangular plaquette can be realised in our system. The magnitude of the flux, denoted $\Phi$, has thus opposite sign for upwards and downwards pointing triangular plaquettes as depicted in figure 3(a) and can be tuned continuously between 0 and $\pi$. 
(a)
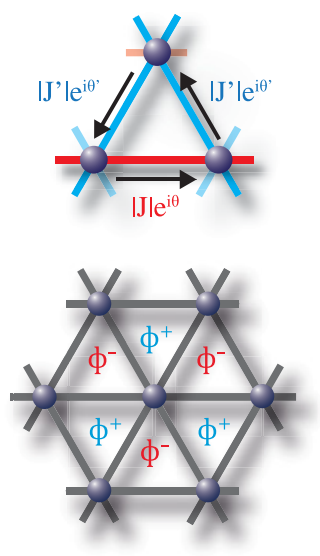

(b)
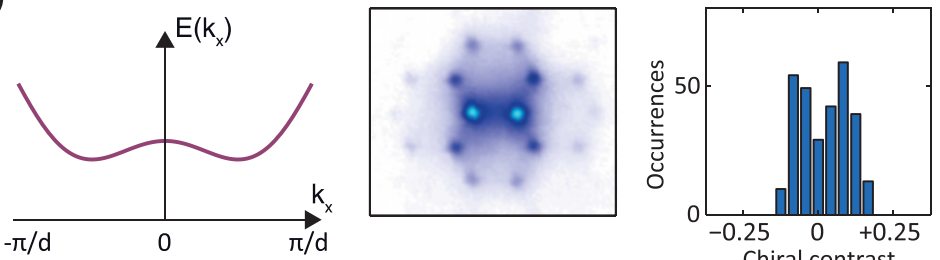

(c)

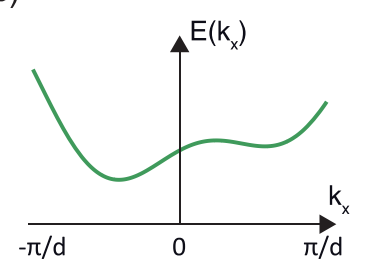

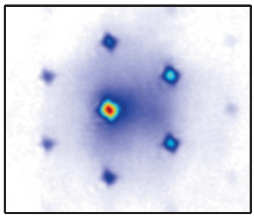

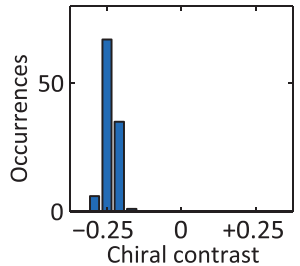

Figure 3. Creation of gauge fluxes in triangular lattices. (a) Complex tunneling elements created by the 2D forcing and resulting staggered magnetic fluxes in a $2 \mathrm{D}$ triangular lattice. (b), (c) Control over the frustration of the system illustrated for $\Phi=\pi$ and $\Phi=0.81 \pi$ respectively. The calculated dispersion relation is ploted along the $x$ axis, demonstrating the control over the frustration of the system. From roughly 100 successive measurements, the summed time-of-flight images and the histograms are extracted. In the degenerate case, the occupation of both ground-states is equiprobable and spontaneous symmetry breaking can be evidenced. For a flux strength of $\Phi=0.81 \pi$, only the global minimum of the band structure is occupied.

As gauge fluxes influence the kinetic part of the Hamiltonian, they strongly modify the single particle dispersion relation, which now reads:

$$
E\left(k_{x}, k_{y}\right)=-2|J| \cos \left(d k_{y}+\theta\right)-2\left|J^{\prime}\right|\left[\cos \left(\frac{\left(k_{x} \sqrt{3}-k_{y}\right) d}{2}+\theta^{\prime}\right)+\cos \left(\frac{\left(k_{x} \sqrt{3}+k_{y}\right) d}{2}-\theta^{\prime}\right)\right] .
$$

For a maxinmal flux strength $|\Phi|=\pi$, frustration is reflected by the presence of two energetically degenerated minima within the first Brillouin zone (see Fig. 3(b)). As shown in Figure 3(c), this degeneracy is lifted for flux amplitudes differing from $\pi$.

\subsection{Spontaneous symmetry breaking}

The presence of non-zero staggered fluxes enlarge the spectrum of the physics that can be investigated with cold atoms in optical lattices. Staggered $\pi$-fluxes introduce a discrete symmetry in the system since the superfluid state may occupy two energetically equivalent quasimomentum states. Frustration leads to fascinating effects, amoung them spontaneous symmetry breaking as already observed in our group [23]. Finding a clean testing ground to experimentally study the process of symmetry breaking is notoriously difficult, as external fluctuations and asymmetries have to be minimised or controlled. The recent achievement of the control over the amplitude of the staggered fluxes allows to investigate this fundamental aspect of frustration in more detail.

In the fully frustrated case, the two degenerate ground states correspond to opposite quasimomentum states along $x$ and can thus be distinguished after a time-of-flight measurement (see Fig. 3(b)). We realised successive measurements of the quasimomentum of the superfluid under the same experimental conditions, which are depicted in a histogram. As an essential result, the system resides in only one of the ground states for each single experimental realisation - the occupation of both momentum states being equiprobable. This is a clear signature for spontaneous symmetry breaking, expected for low energy phases of systems governed by a discrete symmetry. 
By varying the amplitude of the flux, the degeneracy between the ground states can be lifted. Realising the same series of measurement for a flux of amplitude $0.81 \pi$, both the summed time-offlight images and the histogram of Figure 3(c) show that only the global minimum of the single particle dispersion relation is occupied.

\section{CONCLUSION}

Time-periodic driving offers a robust technique to generate gauge fluxes in optical lattices. As reported in these proceedings, our method enables one to continuously control the strength of staggered gauge fluxes in triangular lattices. For maximal staggered $\pi$-fluxes, an additional discrete symmetry impacts the system and leads to the observation of spontaneous symmetry breaking.

As already mentioned, such a spatially homogeneous forcing can not produce finite magnetic fluxes through plaquettes having pairwise parallel bounds as cubic lattices for instance. This restriction can be overcome if a periodically driven superlattice is added. Moreover the presence of the superlattice leads to a flux-rectification over large patches, where strong-field quantum Hall-type physics can be studied [24].

Beyond the engineering of the hopping element, another essential aspect of our scheme is the possibility to address the atomic internal degrees of freedom independently. Here a spin-dependent hexagonal lattice provides a promising system [25]. In such a geometry the interplay of next-nearest neighbour coupling, complex tunneling matrix elements and spin can lead to topological states of matter [24].

The general method presented in these proceedings will facilitate the creation of large sets of gauge fields for various particle classes in optical lattices.

\section{References}

[1] J. Dalibard et al., Rev. Mod. Phys. 83 (2011), 1523.

[2] M. Lewenstein et al., Ultracold Atoms in Optical Lattices: Simulating quantum many-body systems, (Oxford university Press, 2012).

[3] Y. Aharonov and D. Bohm, Phys. Rev. 115 (1959), 485.

[4] J. Struck et al., Phys. Rev. Lett. 108 (2012), 225304.

[5] K. Jiménez-García et al., Phys. Rev. Lett. 108 (2012), 225303.

[6] K. Drese et al., Phys. Rev. Lett. 78 (1997), 2932.

[7] V.V. Ivanov et al., Phys. Rev. Lett. 100 (2008), 043602.

[8] A. Alberti et al., Nature Physics 5 (2009), 547.

[9] C. Sias et al., Phys. Rev. Lett. 100 (2008), 040404.

[10] E. Haller et al., Phys. Rev. Lett. 104 (2010), 200403.

[11] H. Lignier et al., Phys. Rev. Lett. 99 (2007), 220403.

[12] E. Kierig et al., Phys. Rev. Lett. 100 (2008), 190405.

[13] A. Zenesini et al., Phys. Rev. Lett. 102 (2009), 100403.

[14] D. Jaksch et al., Physics 5 (2012), 60.

[15] A. Eckardt et al., Phys. Rev. Lett. 95 (2005), 260404.

[16] A. Eckardt et al., Europhys. Lett. 89 (2010), 10010.

[17] V. Bretin et al., Phys. Rev. Lett. 92 (2004), 050403.

[18] Y.-J. Lin et al., Phys. Rev. Lett. 102 (2009), 130401.

[19] Y.-J. Lin et al., Nature 462 (2009), 628.

[20] M. Aidelsburger et al., Phys. Rev. Lett. 107 (2011), 255301.

[21] J. Dalibard et al., Rev. Mod. Phys. 83 (2011), 1523.

[22] C. Becker et al., New J. Phys. 12 (2010), 065025.

[23] J. Struck et al., Science 333 (2011), 996.

[24] P. Hauke et al., Phys. Rev. Lett. 109 (2012), 145301.

[25] P. Soltan-Panahi et al., Nature Physics 7 (2011), 434. 